\title{
COMPLETIONS OF SEMILATTICES OF CANCELLATIVE SEMIGROUPS
}

\author{
by W. D. BURGESS $\dagger$
}

(Received 15 September, 1978)

Introduction. A semilattice of cancellative semigroups $S$ is a p.o. semigroup with the order relation $a \leq b$ iff $a b=a^{2}$. If $S$ is a strong semilattice of cancellative semigroups (i.e., multiplication in $S$ is given by structure maps $\phi_{e, f}(f \leq e$ in $E)$ ), for each supremumpreserving completion $\bar{E}$ of the semilattice $E$ there is a strong semilattice of cancellative semigroups $T$ over $\bar{E}$ which is a supremum-preserving completion of $S$ in $\leq$. Given $\bar{E}, T$ is constructed directly. In this paper it is shown that multiplication by an element of $S$ distributes over suprema in $\leq$ if $E$ has this property (called strong distributivity). Next it is shown that the completion construction also applies to a semilattice of cancellative semigroups which is not strong if $S$ is commutative and $\bar{E}$ is strongly distributive. Finally, it is shown that for semilattices of cancellative monoids a completion is completely determined, up to isomorphism over $S$, by completions of $E$.

We begin by noting that if $S$ is a semilattice of cancellative semigroups $S_{e}(e \in E)$ then there are three particular ways of defining an order relation on $S$, namely

and

$$
a \leq_{1} b \Leftrightarrow a b=a^{2}, \quad a \leq_{2} b \Leftrightarrow b a=a^{2}
$$

$$
a \leq_{3} b \Leftrightarrow a s b=b s a=a s a \text { for all } s \in S
$$

(see [5] and [10]). These all coincide in this case. For if $a \in S_{e}, b \in S_{f}$ and $a \leq_{1} b$ then $a b=a^{2}$ (giving $e \leq f$ ), so that $a b a=a^{3}$ and $b a=a^{2}$, since $S_{e}$ is cancellative. Hence $\leq_{1}$ and $\leq_{2}$ coincide. If $a \leq_{3} b$ then $a^{2} b=a^{3}$ giving $a \leq_{1} b$, while if $a \leq_{1} b$ and $s \in S$, the equation

$$
\text { asbasa }=\text { asaasa }
$$

and cancellation give the remaining equivalence. Necessary and sufficient conditions for these relations to be order relations are found in [5] and [10]. This is the case for semilattices of cancellative semigroups.

In the case of inverse semigroups whose idempotents are central, this order coincides with the natural order for an inverse semigroup [6, p. 40]. In particular this applies to semilattices.

The order relation on $S$ makes $S$ into a p.o. semigroup [5, Proposition 3] and the relation is called Abian's order. A subset $X$ of $S$ can have an upper bound in $S$ only if it is boundable, i.e., for $x, y \in X, x y^{2}=x^{2} y$. A semigroup $S$ is complete if every boundable set in $S$ has a supremum. An embedding $S \subset T$ of semigroups is a completion if (i) $T$ is a semilattice of cancellative semigroups, (ii) $T$ is complete and (iii) every element of $T$ is the supremum of some boundable set in $S$. We shall be dealing with completions such that the inclusion $S \subset T$ preserves suprema which exist in $S$.

$\dagger$ This research was partially supported by Grant A 7539 of the National Research Council.

Glasgow Math. J. 21 (1980) 29-37. 
Finally, if $S=\bigcup_{E} S_{e}$ is a strong semilattice of cancellative semigroups (i.e., for $f \leq e$ in $E$ there are homomorphisms $\phi_{e, f}: S_{e} \rightarrow S_{f}$ such that for $a \in S_{e}, b \in S_{f}, a b=$ $\left.\phi_{e, e f}(a) \phi_{f, e f}(b)\right)$ then a set $X \subset S$ is boundable if and only if for $x, y \in X, x \in S_{e}, y \in S_{f}$ then $\phi_{e, e f}(x)=\phi_{f, e f}(y)$. Note that distinct elements of a boundable set are in distinct cancellative parts of $S$.

1. The completion of a strong semilattice of cancellative semigroups. Throughout this part let $S$ be a semigroup which is a semilattice $E$ of cancellative semigroups $S_{e}$ $(e \in E)$, where multiplication in $S$ is given by structure maps $\phi_{e, f}: S_{e} \rightarrow S_{f}$ for $e, f \in E, f \leq e$.

The construction of a completion $T$ for $S$ is done by directly constructing $T$ as a lattice of cancellative semigroups. This construction owes something to the construction of semigroups of quotients of semilattices of groups as found in [9] and [11], but here a completion of $E$ is at the base of it all and the cancellative components need not be groups.

EXAMPLEs. Consider the following lattices of groups.
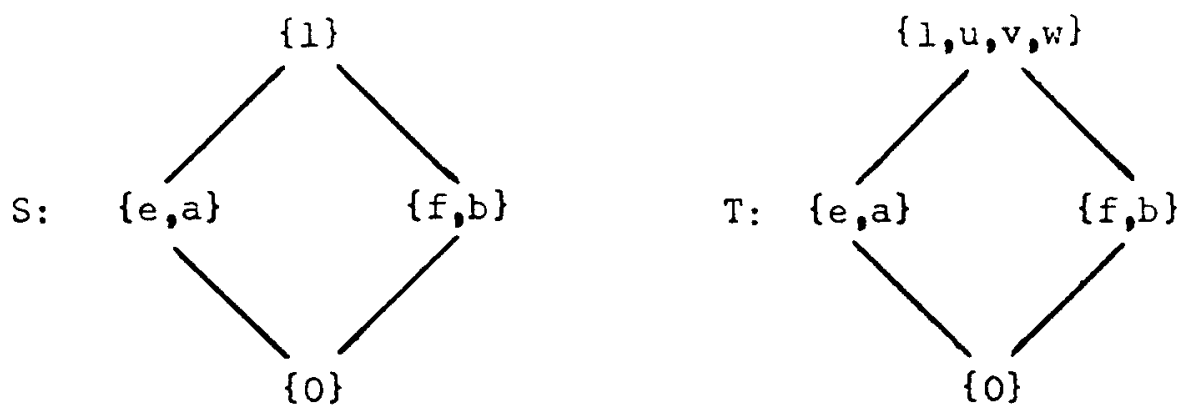

where, in both, $e^{2}=e, f^{2}=f$. In $T, \operatorname{ker} \phi_{1, e}=\{1, u\}$, $\operatorname{ker} \phi_{1, f}=\{1, v\}$. The boundable sets of $S$ are: the singletons, $\{e, f\},\{e, b\}\{a, f\},\{a, b\}$ and these four with 0 added and $\{1, e, f, 0\}$. We have that $S$ is not complete since, for example, $\{a, b\}$ has no upper bound. However $T$ is a completion of $S$ with the obvious embedding. This is a model for the general construction.

The semilattice $E$ can be completed in various ways, $E \subseteq \bar{E}, \bar{E}$ a complete lattice. In particular we may take $\bar{E}$ to be the Dedekind-MacNeille completion where the element $f \in \bar{E}$ corresponds to the subset $A=\{e \in E \mid e \leq f\}$ of $E$ (see [12, p. 44]). The embedding $E \subseteq \bar{E}$ preserves all suprema which exist in $E$. The completion to be constructed will be a lattice of cancellative semigroups $T=\bigcup_{E} T_{f}$. (In order to obtain the theorem below, any supremum-preserving completion of $E$ will suffice, the particular one being mentioned only for concreteness.) The construction of $T$ and the verification of its properties will be done in six steps. In order to establish notation for the remainder of the article, to $f \in \bar{E}$ we make correspond a subset of $E$ as follows: if $f=\sup \{e \in E \mid e<f\}$ then we let $A=\{e \in E \mid e<f\}$ (this occurs if $f \in \bar{E} \backslash E$ and for some elements of $E$ ); if $f \neq \sup \{e \in$ $E \mid e<f\}$ we let $A=\{e \in E \mid e \leq f\}$ (this can only occur for some elements of $E$, for 
example $0, e, f$ in the preceding example, but not 1 ). Whichever case occurs $A$ will be called the subset of $E$ corresponding to $f$.

STEP 1. For $f \in \bar{E}$, let $A$ be the corresponding subset of $E$. Define $T_{f}$ to be the inverse limit of the system

$$
\left\{S_{e} ; \phi_{e, e^{\prime}}, e, e^{\prime} \in A, e^{\prime} \leq e\right\}
$$

(see [8, p. 291]); that is, $T_{f}$ is the subsemigroup of $\prod_{A} S_{e}$ consisting of the elements $\left(x_{e}\right)_{A}$ such that if $e^{\prime} \leq e\left(e, e^{\prime} \in A\right)$, then $\phi_{e, e^{\prime}}\left(x_{e}\right)=x_{e^{\prime}}$. The result is clearly cancellative and if the $S_{e}(e \in A)$ are groups, so is $T_{f}$. Note that $T_{f}$ could be empty although not in the case where each $S_{e}(e \in A)$ contains an idempotent. Also if $f \in E$ and $A=\{e \in E \mid e \leq f\}$ then $T_{f}=S_{f}$.

The elements of $T_{f}$ are precisely the boundable sets $X$ of $S$ such that

(i) if $s \leq x$ for some $s \in S, x \in X$, then $s \in X$, and

(ii) $\left\{e \in E \mid x \in S_{e}\right.$ for some $\left.x \in X\right\}=A$.

STEP 2. Put $T=\bigcup_{\bar{E}} T_{f}$ and define multiplication via structure maps as follows. If $f, f^{\prime} \in \bar{E}, f^{\prime} \leq f$ with corresponding subsets of $E, B \subseteq A$, then define $\psi_{f, f^{\prime}}: T_{f} \rightarrow T_{f^{\prime}}$ by $\psi_{f, f^{\prime}}\left(\left(x_{e}\right)_{A}\right)=\left(x_{e}\right)_{B}$, the restriction of $\left(x_{e}\right)_{A} \in T_{f} \subseteq \prod_{A} S_{e}$ to $B$. Then in general if $f, f^{\prime} \in \bar{E}$ have corresponding subsets $A$ and $B$ of $E$, respectively,

$$
\left(x_{e}\right)_{A}\left(y_{e^{\prime}}\right)_{B}=\psi_{f^{\prime}, f f^{\prime}}\left(\left(y_{e^{\prime}}\right)_{B}\right) \text {. }
$$

Abian's order is defined on $T$.

STEP 3. The embedding of $S \subseteq T$ is as follows. If $e \in E$ then $e \in \bar{E}$ and we assign to $x \in S_{e}$ the element $\left(x_{e^{\prime}}\right)_{A}$ where $A$ is the subset of $E$ corresponding to $e$ and for $e^{\prime} \in A$, $x_{e^{\prime}}=\phi_{e, e^{\prime}}(x)$. This embedding is clearly order-preserving.

STEP 4. Every element of $T$ is the supremum of a subset of $S$. Consider $x=\left(x_{e}\right)_{A} \in T_{f}$ then $\left(x_{e}\right)_{A}=\sup \left\{x_{e} \mid e \in A\right\}$. Indeed, for $e^{\prime} \in A$,

$$
x x_{e^{\prime}}=\psi_{f, e^{\prime}}\left(\left(x_{e}\right)_{\mathrm{A}}\right) x_{e^{\prime}}=x_{e^{\prime}}^{2}
$$

Thus $y$ is an upper bound of $X=\left\{x_{e} \mid e \in A\right\}$. Suppose that $y=\left(y_{e}\right)_{B} \in T_{f^{\prime}}$ is an upper bound of $X$. Then $\left(y_{e}\right)_{B} x_{e^{\prime}}=x_{e^{\prime}}^{2}$, showing that $e^{\prime} \leq f^{\prime}$ for all $e^{\prime} \in A$. Hence, $A \subseteq B$ and $f \leq f^{\prime}$. By the cancellation property, for $e \in A, y_{e}=x_{e}$ so that $\psi_{f^{\prime}, f}(y)=x$ and so $x y=x^{2}$, giving the result.

SteP 5. The semigroup $T$ is complete. Let $X=\left\{\left(x_{e}^{\alpha}\right)_{A_{\alpha}} \mid \alpha \in \Lambda\right\}$ be a boundable set in $T$. We may assume that if $t \in T, x \in X$ and $t \leq x$ then $t \in X$. We have that for $\left(x_{e}^{\alpha}\right)_{A_{\alpha}}$ and $\left(x_{e}^{\beta}\right)_{A_{\beta}}$ in $X$,

$$
\left(x_{e}^{\alpha}\right)_{A_{\alpha}}^{2}\left(x_{e}^{\beta}\right)_{A_{\beta}}=\left(x_{e}^{\alpha}\right)_{A_{\alpha}}\left(x_{e}^{\beta}\right)_{A_{\beta}}^{2}
$$

Calculating we get

$$
\left(x_{e}^{\alpha}\right)_{A_{\alpha}}^{2}\left(x_{e}^{\beta}\right)_{A_{\beta}}=\left(\left(x_{e}^{\alpha}\right)^{2} x_{e}^{\beta}\right)_{A_{\gamma}}=\left(x_{e}^{\alpha}\left(x_{e}^{\beta}\right)^{2}\right)_{A_{\gamma}}
$$


where if $A_{\alpha}$ corresponds to $f_{\alpha}$ and $A_{\beta}$ to $f_{\beta}$ then $A_{\gamma}$ corresponds to $f_{\alpha} f_{\beta}$; thus $A_{\gamma}=A_{\alpha} \cap A_{\beta}$. Hence for all $e \in A_{\gamma}, x_{e}^{\alpha}=x_{e}^{\beta}$. Put

$$
U=\left\{x_{e}^{\alpha} \mid e \in A_{\alpha}, \alpha \in \Lambda\right\} \subseteq S .
$$

Then

$$
\begin{aligned}
\left(x_{e}^{\alpha}\right)^{2} x_{e^{\prime}}^{\beta} & =\phi_{e, e e^{\prime}}\left(\left(x_{e}^{\alpha}\right)^{2}\right) \phi_{e^{\prime}, e e^{\prime}}\left(x_{e^{\prime}}^{\beta}\right) \\
& =\left(x_{e e^{\prime}}^{\alpha}\right)^{2} x_{e e^{\prime}}^{\beta}=x_{e e^{\prime}}^{\alpha}\left(x_{e e^{\prime}}^{\beta}\right)^{2} \\
& =x_{e}^{\alpha}\left(x_{e}^{\beta}\right)^{2} .
\end{aligned}
$$

Hence $U$ is boundable.

Put $A=\left\{e \in E \mid x_{e}^{\alpha} \in S_{e}\right.$ for some $\left.x_{e}^{\alpha} \in U\right\}$. This set has a supremum $f$ in $\bar{E}$ and $f=\sup \left(\sup A_{\alpha}\right)(\operatorname{see}[1, \mathrm{p} .53])$; hence if $e \in E, e \leq f$ then $e \leq \sup A_{\alpha}$ for some $e \in A$ anc thus $e \in A_{\alpha}$. Now put $x=\left(x_{e}\right)_{A}$ where $x_{e}$ is any $x_{e}^{\alpha}(\alpha \in \Lambda)$. This is well-defined, since it $e \in A_{\alpha} \cap A_{\beta}$ then $x_{e}^{\alpha}=x_{e}^{\beta}$. We claim that $x=\sup X$.

For $\left(x_{e}^{\alpha}\right)_{A_{\alpha}} \in X$ we have

$$
\left(x_{e}\right)_{A}\left(x_{e^{\prime}}^{\alpha}\right)_{A_{\alpha}}=\left(x_{e^{\prime}} x_{e^{\prime}}^{\alpha}\right)_{A_{\alpha}},
$$

since $A_{\alpha} \subseteq A$; hence for $e^{\prime} \in A_{\alpha}, x_{e^{\prime}}=x_{e^{\prime}}^{\alpha}$ and we conclude that

$$
x\left(x_{e^{\prime}}^{\alpha}\right)_{\mathrm{A}_{\alpha}}=\left(x_{e^{\prime}}^{\alpha}\right)_{\mathrm{A}_{\alpha}}^{2} .
$$

Hence $x$ is an upper bound. But $x=\sup U$ and every $x_{e}^{\alpha} \in U$ is below an element of $:$ (indeed if $x_{e}^{\alpha} \in\left\{x_{e^{\prime}}^{\alpha}\right\}_{A_{\alpha}}$ then $x_{e}^{\alpha} \leq\left(x_{e^{\prime}}^{\alpha}\right)_{A_{\alpha}}$ by the nature of the embedding $S \subseteq T$ ). Henc $x=\sup X$.

STEP 6. The embedding $S \subseteq T$ preserves all suprema which exist in $S$. Let $s=\sup$; ( $X$ a subset of $S, s \in S$ ). Then if $A=\left\{e^{\prime} \mid x \in S_{e^{\prime}}\right.$ for some $\left.x \in X\right\}$ and $s \in S_{e}$, it follows tha $e=\sup A$. If $g \leq e$ is another upper bound of $A$ in $E$, it is readily seen that $y=\phi_{e, g}(x) \mathrm{j}$ an upper bound of $X$ and that $y \leq x$. Hence $y=x$ and $e=g$. But $e=\sup A$ in $\vec{E}$ as we and the boundable set $X$ has a supremum $t$ in $T_{e}$, and hence $t \leq s$. But $T_{e}$ is cancellative so $s=t$.

This completes the construction, giving the following theorem; its corollary follow from it and the remark in Step 1.

THEOREM 1. Let $S$ be a semigroup with decomposition $S=\bigcup_{E} S_{e}$, where $E$ is semilattice and the $S_{e}$ are cancellative. Suppose further that multiplication in $S$ is given $b$ structure maps $\phi_{e, e^{\prime}}: S_{e} \rightarrow S_{e^{\prime}}$ for $e^{\prime} \leq e$ in $E$. Then $S$ has a completion in Abian's order i where $T$ is a semigroup of the same type as $S$ and the inclusion $S \subseteq T$ preserves suprema fror $S$.

Corollary 2. Let $S$ be a semilattice of groups. Then $S$ has a completion $T$ in Abian order, where $T$ is a lattice of groups. 
The completion of a semigroup is not unique (unlike the case for rings [2, Theorem 12]) since even a lattice may be completed in several non-isomorphic ways. Uniqueness will be discussed further in part 4 below. Theorem 1 does yield an internal characterization of complete semigroups (of the type being studied here). The proof is clear from the proof of Theorem 1.

Proposirion 3. Let $S$ be a semigroup with decomposition $S=\bigcup_{E} S_{e}$, where $E$ is a semilattice, the $S_{e}$ are cancellative and the multiplication in $S$ is given by structure maps $\phi_{e, e^{\prime}}: S_{e} \rightarrow S_{e^{\prime}}\left(e^{\prime} \leq e\right.$ in $\left.E\right)$. Then $S$ is complete if and only if (i) $E$ is a complete lattice, (ii) if $f \in E$ is such that $f=\sup A$ where $A=\{e \in E \mid e<f\}$ then $S_{f}=\lim _{-A}\left\{S_{e} ; \phi_{e, e}\right\}$ and (iii) if $e^{\prime} \leq e$ in $E$ then $\phi_{e . e^{\prime}}$ is the homomorphism induced by the universal property of inverse limits.

ExAmple. Let $E$ be a semilattice with 0 such that $e f=0$ for all $e \neq f$. Then with $S_{0}=\{0\}$ and $S_{e}$ arbitrary $(e \neq 0)$, a semigroup $S=\bigcup_{E} S_{e}$ can be formed. By adjoining an

element 1 to $E$ we get a completion $\bar{E}$. Clearly $T_{1}=\prod_{E} S_{e}$ and $T_{e}=S_{e}$ for all $e \in E$. Here $\bar{E}$ is the Dedekind-MacNeille completion of $E$. Using the same $E$ we can also form the ideal completion $F$ of $E$, which in this case is supremum-preserving (it is not always [7]); $F$ is the set of all subsets of $E$ which contain 0 . For $U \in F, T_{U}=\prod_{U} S_{e}$. These two completions are clearly not isomorphic.

2. Distributivity. In the case of semiprime rings, Abian's order and Conrad's order satisfy an infinite distributivity: if $R$ is a semiprime ring and if $x=\sup X, a \in R$ then $\sup a X=a x$ and $\sup X a=x a$ ([4, Corollary 3]). For semigroups this is false since there are lattices which are not distributive. However, for the type of semigroups we have been studying, distributivity will be seen to be a property of the underlying semilattice. Let us say that a semilattice $L$ is strongly distributive if for any subset $X$ of $L$ and $e \in L$, if $\sup X$ exists then $\sup e X=e(\sup X)$.

Proposirion 4. Let $S$ be a semigroup with a decomposition $S=\bigcup_{E} S_{e}$, where $E$ is a semilattice, the $S_{e}$ are cancellative and multiplication in $S$ is given by structure maps $\phi_{e, e^{\prime}}: S_{e} \rightarrow S_{e^{\prime}}\left(e^{\prime} \leq e\right.$ in $\left.E\right)$. Suppose that $E$ is strongly distributive. Then for any boundable set $X$ of $S$ and any $a \in S, \sup a X=a(\sup X)$ and $\sup X a=(\sup X) a$ if $\sup X$ exists.

Proof. Let $y=\sup X$. If $A=\left\{e \in E \mid x \in X \cap S_{e}\right.$ for some $\left.x\right\}$, then clearly if $y \in S_{f}$ we have $f=\sup A$. Let $a \in S_{\mathrm{g}}$ and consider

$$
\begin{aligned}
\operatorname{ayax} & =\phi_{\mathrm{g}, \mathrm{ge}}(a) \phi_{f, g e}(y) \phi_{\mathrm{g}, \mathrm{ge}}(a) \phi_{e, g e}(x) \\
& =\phi_{g, g e}(a) \phi_{e, g e}(x) \phi_{g, g e}(a) \phi_{e, g e}(x) \\
& =\operatorname{axax} \text { for } x \in X \cap S_{e} .
\end{aligned}
$$

Hence $a y$ is an upper bound for $a X$. Let $u \in S_{h}$ be another upper bound for $a X$. Since $h$ is 
an upper bound for $g A$,

$$
h \geq \sup g A=g(\sup A)=g f .
$$

It follows that $\phi_{h, g f}(u)$ is an upper bound of $a X$ in $S_{g f}$. By cancellation, $\phi_{h, g f}(u)=a y$ and $a y \leq u$.

An analogous statement for inverse semigroups is [13, Lemma 1.13].

Note that strong distributivity for semilattices and, more generally, for semigroups with Abian's order implies the following distributive property: if $S$ is a strong semilattice of cancellative semigroups such that for $s \in S$ and a boundable set $X, \sup s X=s(\sup X)$ if either exists, then for boundable sets $X$ and $Y$ we get that $X Y=\{x y \mid x \in X, y \in Y\}$ is boundable and $\sup X Y=(\sup X)(\sup Y)$ if either side exists.

3. A generalization. In this section we attempt to construct a completion of a semilattice of cancellative semigroups where there are no structure maps available. It will be necessary to impose supplementary conditions on the cancellative semigroups and on the semilattice.

THEOREM 5. Let $S$ be a commutative semigroup which is a semilattice $\bigcup_{E} S_{e}$ of cancellative semigroups. Assume further that $E$ has a supremum-preserving completion $\bar{E}$ which is strongly distributive. Then $S$ has a supremum-preserving completion.

Proof. We first construct for each $e \in E$ the group $G_{e}$ of fractions of $S_{e}$. For $a b^{-1} \in G_{e}$ and $c d^{-1} \in G_{e^{\prime}}$ define $a b^{-1} \cdot c d^{-1}=a c(b d)^{-1} \in G_{e e^{\prime}}$. Let $G=\bigcup_{E} G_{e}$ with the indicated multiplication; it is a semigroup of the type studied in Part 1 . Let $T=\bigcup_{E} T_{f}$ be the completion of $G$ as constructed in Theorem 1 .

For $f \in \bar{E}$ let $A$ be the corresponding subset of $E$ (see Part 1 for notation) and recall that an element of $T_{f}$ has the form $\left(x_{e}\right)_{A}$ where if $e^{\prime} \leq e$ in $A$ then $\phi_{e, e^{\prime}}\left(x_{e}\right)=x_{e^{\prime}}$. Put

$$
U_{f}=\left\{\left(x_{e}\right)_{A} \in T_{f} \mid \text { for some } B \subseteq A, \quad \sup B=\sup A=f, \quad x_{e} \in S_{e} \text { for all } e \in B\right\}
$$

These are elements of $T_{f}$ which are, in a sense, "almost everywhere" in $S$. We put $U=\bigcup_{E} U_{f}$ and we shall show that $U$ is the desired completion. Note that, as remarked in Part 2, if $\bar{E}$ is strongly distributive and $A, B \subseteq \bar{E}$ then $\sup A B=(\sup A)(\sup B)$; indeed

$$
\begin{aligned}
\sup A B & =\sup _{A}(\sup a B)=\sup _{A}(a \sup B) \\
& =\sup (A \sup B)=(\sup A)(\sup B) .
\end{aligned}
$$

Firstly, $U$ is a subsemigroup of $T$. Let $\left(x_{e}\right)_{A} \in U_{f}$ and $\left(y_{e}\right)_{A^{\prime}} \in U_{f^{\prime}}$ where $A$ and $A^{\prime}$ are the subsets of $E$ corresponding to $f$ and $f^{\prime}$ respectively and for some $B \subseteq A, \sup B=f$, $x_{e} \in S_{e}$ for all $e \in B$ and for some $B^{\prime} \subseteq A^{\prime}, \sup B^{\prime}=f^{\prime}, y_{e} \in S_{e}$ for all $e \in B^{\prime}$. Then

$$
\left(x_{e}\right)_{A} \cdot\left(y_{e}\right)_{A^{\prime}}=\left(x_{e} y_{e}\right)_{A^{\prime}}
$$

(of course $\left.A A^{\prime}=\left\{e \in E \mid e \leq f f^{\prime}\right\}\right)$. But $\sup B B^{\prime}=(\sup B)\left(\sup B^{\prime}\right)=f^{\prime}$ (by hypothesis) and $x_{e} y_{e} \in S_{e}$ for all $e \in B B^{\prime}$. 
Lemma. If $\left(x_{e}\right)_{A} \in T_{f}, A$ is the subset of $E$ corresponding to $f$ and $B \subseteq A$ is such that $\sup B=f$ then $\sup \left\{x_{e} \mid e \in A\right\}=\sup \left\{x_{e} \mid e \in B\right\}$.

Proof. Let $x=\sup \left\{x_{e} \mid e \in A\right\}, y=\sup \left\{x_{e} \mid e \in B\right\}$. Clearly both $x$ and $y$ are in $T_{f}$ and $y \leq x$. This gives the equality.

Corollary. If $\left(x_{e}\right)_{A},\left(y_{e}\right)_{A} \in T_{f}$ and for some $B \subseteq A$, with $\sup B=f, x_{e}=y_{e}$ for all $e \in B$ then $\left(x_{e}\right)_{A}=\left(y_{e}\right)_{A}$.

Proof. By the lemma, $\sup \left\{x_{e} \mid e \in B\right\}=\left(x_{e}\right)_{\mathrm{A}}=\left(y_{e}\right)_{\mathrm{A}}$.

Returning to the theorem, we must show that $U$ is complete; it will follow that $U$ is a completion of $S$, since if $\left(x_{e}\right)_{A} \in U_{f}$ and $B \subseteq A$ with $\sup B=f$ and $x_{e} \in S_{e}$ for all $e \in B$ then the lemma shows that $\left(x_{e}\right)_{\mathrm{A}}=\sup \left\{x_{e} \mid e \in \dot{B}\right\}$, the supremum of a subset of $S$.

Let $X=\left\{\left(x_{e}^{\alpha}\right)_{A_{\alpha}} \mid \alpha \in \Lambda\right\}$ be a boundable set from $U$, where $A_{\alpha} \subseteq E$ corresponds to $f_{\alpha}$, $B_{\alpha} \subseteq A_{\alpha}$, sup $B_{\alpha}=f_{\alpha}$ and $x_{e}^{\alpha} \in S_{e}$ for all $e \in B_{\alpha}$. Put $x=\sup X$, an element of $T$. It will be shown that $x \in U$. Since $X$ is boundable, for $e \in A_{\alpha} \cap A_{\beta}=A_{\alpha} A_{\beta}$ we have $x_{e}^{\alpha}=x_{e}^{\beta}$. Let

$$
Y=\left\{x_{e} \mid x_{e}=x_{e}^{\alpha} \quad \text { for some } e \in \bigcup_{\Lambda} A_{\alpha} \text { and some } \alpha \in \Lambda\right\} \text {. }
$$

As was shown in Theorem 1 , Step $5, Y$ is boundable with the same supremum as $X$. Now consider $\bigcup B_{\alpha} \subseteq \bigcup A_{\alpha}$. We have

$$
\begin{aligned}
\sup \bigcup B_{\alpha} & =\sup \left\{\sup B_{\alpha} \mid \alpha \in \Lambda\right\}=\sup \left\{f_{\alpha} \mid \alpha \in \Lambda\right\} \\
& =\sup \left\{\sup A_{\alpha} \mid \alpha \in \Lambda\right\}=\sup \bigcup A_{\alpha}=f .
\end{aligned}
$$

Hence $x \in U_{f}$.

It would be desirable to weaken the conditions on Theorem 6 to those of Theorem 1 .

4. Uniqueness of completions. It has already been mentioned that completions are not unique since semilattices may have non-isomorphic completions. However, in the case of a semilattice of monoids, it will be shown that there is, up to isomorphism over $S$, one supremum-preserving completion of $S$, which is a semilattice of cancellative semigroups, for each isomorphism class of supremum-preserving completions of the underlying semilattice $E$.

THEOREM 6. Let $S=\bigcup_{E} S_{e}$ be a semilattice of cancellative monoids and let $U=\bigcup_{F} U_{f}$ be a semilattice of cancellative semigroups which is a supremum-preserving completion of $S$. Then (i) each $U_{f}$ is a monoid, (ii) $F$ is a supremum-preserving completion of $E$, (iii) $U$ is isomorphic over $S$ to the completion constructed over $F$ in Theorem 1.

Proof. $E$ is contained in $F$ as semilattices, for if $e \in E \subseteq S$ and $e \in U_{f}$ then for $s \in S_{e}$, $s=s e$. It follows that $s \in U_{f}$. Further if $e, e^{\prime} \in E$ with $e \in U_{f}, e^{\prime} \in U_{f^{\prime}}$ then $e e^{\prime} \in U_{f^{\prime \prime}}$. Hence if $e \in U_{f}, e$ may be identified with $f$. Further, $F$ is a supremum-preserving completion of $E$. 
Let $u \in U_{f}, u=\sup X$ for some $X \subseteq S$. Put

$$
A=\left\{e \in E \mid x \in S_{e} \text { for some } x \in X\right\} .
$$

Now for $x \in X, x \in S_{e}, u x=x^{2} \in S_{e} \subseteq U_{e}$, so that $e \leq f$. Since $U$ is complete, the boundable set $A$ has a supremum $g$ in $U$. Now $g$ is an idempotent, since $g^{2} e=g e=e$ for all $e \in A$, which shows that $g^{2}$ is also an upper bound of $A$; hence $g \leq g^{2}$ giving $g^{3}=g^{2}$ in a cancellative semigroup. Thus $g=g^{2}$. We also have

$$
g u x=g x^{2}=g e x^{2}=e x^{2}=x^{2}
$$

for $x \in X \cap S_{e}$, and so $g u \geq u$. From this $g u^{2}=u^{2}$, showing that $g \in U_{f}$. We may identify $g$ with $f \in F$. (ii).

It follows that each $U_{f}$ is a monoid. By Proposition $3, F$ is complete, giving (i) and

Now let $f \in F$ with corresponding set $A \subseteq E$. Each $u \in U_{f}$ is the supremum of some $X \subseteq S$. Let

$$
B=\left\{e \in E \mid x \in X \cap S_{e} \text { for some } x\right\} .
$$

Clearly $B \subseteq A$ and $\sup B=\sup A=f$. Further, if $e \in A$ there is $e^{\prime} \in B$ with $e^{\prime} \geq e$, from which it follows that $u e=u e^{\prime} e$. But if $x \in X \cap S_{e^{\prime}}$, then $u \geq x$ implies that $u e^{\prime}=x$. Hence $u e=x e \in S$. Thus multiplication by $e \in A$ gives a homomorphism $\tau_{e}: U_{f} \rightarrow S_{e}$. Let $T_{f}$ $=\lim _{\leftarrow A}\left\{S_{e} ; \phi_{e, e}\right\}$ (as in Part 1). The homomorphisms $\tau_{e}$ induce a homomorphism $\tau: U_{f} \rightarrow T_{f}$ by the universal property of inverse limits. This is readily seen to be an isomorphism. Further, for $f, f^{\prime} \in F, f^{\prime} \leq f$, multiplication by $f^{\prime}$ gives $U_{f} \rightarrow U_{f^{\prime}}$, which is precisely the induced homomorphism $\psi_{f . f^{\prime}}$ of Theorem 1 . Hence $U$ is isomorphic to $T$ constructed as in Theorem 1 over $F$ and the isomorphism leaves elements of $S$ fixed.

It would be desirable to be able to get this uniqueness result for any strong semilattice of cancellative semigroups.

If the semilattice $E$ is a Boolean algebra then there is only one completion (the Dedekind-MacNeille) and it is strongly distributive. Hence if $R$ is a strongly regular ring then the completion of its multiplicative semigroup is unique; it is based on the completion of $B(R)$, the Boolean algebra of idempotents. This completion is the multiplicative semigroup of the completion of $R$ as a ring which is, in this case, the complete ring of quotients, $Q(R)$ (see [2, Theorem 14] and [3, Theorem 5]). More generally, if $R$ is a reduced p.p. ring (a ring with no non-zero idempotents in which the annihilator of each element is generated by an idempotent; in a reduced ring all idempotents are central and left and right annihilators coincide) then the multiplicative semigroup is a Boolean algebra of cancellative semigroups. Indeed for $e=e^{2} \in R$, put

$$
R_{e}=\left\{r \in R \mid r e=r \text { and if for some } f=f^{2}, r f=r \text { then } e \leq f\right\} .
$$

Now if $r, s, t \in R_{e}$, and $r s=r t$ we get $s-t \in$ Ann $r=g R$ for some $g=g^{2}$. Thus $r(1-g)=r$ and $e \leq 1-g$ giving $e g=0$ and

$$
s-t=g(s-t)=g(e s-e t)=0,
$$


showing that $s=t$. Further, $R=\bigcup_{B(R)} R_{e}$. Let $r \in R$; then Ann $r=e R$ for some $e \in B(R)$ and $r(1-e)=r$. If $r f=r$ for $f \in B(R)$ then $r(1-f)=0$ and $1-f \in e R$, giving $1-e \leq f$. Hence $r \in R_{1-e}$.

Now if $R$ is commutative p.p. ring, it has a completion in Abian's order, call it $C(R)$, and $B(C(R))$ is the Dedekind-MacNeille completion of $B(R)([3$, Theorem 11]). We have shown the following:

Proposition 7. Let $R$ be a commutative p.p. ring. Then there is a unique supremumpreserving completion of the multiplicative semigroup of $R$. It is the multiplicative semigroup of the completion of the ring $R$.

\section{REFERENCES}

1. G. Birkhoff, Lattice theory (Amer. Math. Soc., 1967).

2. W. D. Burgess and R. Raphael, Abian's order relation and orthogonal completeness for reduced rings, Pacific J. Math. 54 (1974), 55-64.

3. W. D. Burgess and R. Raphael, Complete and orthogonally complete rings, Canad. J. Math. 27 (1975), 884-892.

4. W. D. Burgess and R. Raphael, Sur deux notions de complétude dans les anneaux semipremiers, C.R. Acad. Sci. Paris 283 (1976), 927-929.

5. W. D. Burgess and R. Raphael, On Conrad's partial order relation on semiprime rings and on semigroups, Semigroup Forum 16 (1978), 133-140.

6. A. H. Clifford and G. B. Preston, The algebraic theory of semigroups, Vol. II, Math. Surveys of the Amer. Math. Soc. 7 (Providence, R.I., 1967).

7. P. Crawley, Regular embeddings which preserve lattice structure, Proc. Amer. Math. Soc. 13 (1962), 35-47.

8. J. K. Goldhaber and G. Ehrlich, Algebra (Collier-Macmillan, London, 1970).

9. C. V. Hinkle Jr., Semigroups of right quotients of a semigroup which is a semilattice of groups, J. Algebra 31 (1974), 276-286.

10. C. S. Johnson and F. R. McMorris, Abian's order for semigroups, Semigroup Forum 16 (1978), 147-152.

11. F. R. McMorris, The quotient semigroup of a semigroup that is a semilattice of groups, Glasgow Math. J. 12 (1971), 18-23. 1966).

12. J. Lambek, Lectures on rings and modules (Ginn-Blaisdell, Waltham, Toronio, London,

13. B. M. Schein, Completions, translational hulls and ideal extensions of inverse semigroups, Czechoslovak Math. J. 23 (1973), 575-610.

UNIVERSITY OF OTTAWA

Ottawa, Canada K1N 6N5 